\title{
Quality of Life during Treatment with Lenvatinib for Thyroid Cancer: The Patients' Perspective beyond the Medical Evaluation
}

\author{
Alice Nervo ${ }^{a} \quad$ Alberto Ragni $^{a} \quad$ Alessandro Piovesan ${ }^{a}$ Valentina Marica ${ }^{a}$ \\ Enrica Migliore $^{b}$ Marco Gallo ${ }^{a}$ Emanuela Arvat ${ }^{a}$ \\ a Oncological Endocrinology Unit, Department of Medical Sciences, University of Turin, Città della Salute e della \\ Scienza Hospital, Turin, Italy; ${ }^{b}$ Cancer Epidemiology Unit, Department of Medical Sciences, University of Turin, \\ Città della Salute e della Scienza Hospital, Turin, Italy
}

\section{Keywords}

Patient-reported outcome · Health-related quality of life ·

Tyrosine kinase inhibitors · Thyroid cancer

\begin{abstract}
Background: Lenvatinib (LEN) has shown great efficacy but important toxicity in patients with advanced radioactive iodine-refractory (RAI-R) thyroid cancer (TC); a focused evaluation of its impact on patients' quality of life (QoL) is still lacking. Our prospective study investigated the impact of this drug on QoL in a group of RAI-R TC patients treated at our centre. Methods: All patients filled in two questionnaires before and during treatment: (1) the Patient-Reported Outcomes version of the Common Terminology Criteria for Adverse Events adapted questionnaire, assessing prevalence, severity, and/or interference on daily activities of the most frequent symptomatic adverse events (AEs) reported in previous clinical trials during therapy with LEN, and (2) the European Quality of Life 5 Dimensions 3 Levels (EQ-5D-3L) questionnaire, providing the EQ-5D index and the EQ visual analogue scale (EQ-VAS). Data from baseline questionnaires were compared with those obtained 3, 6, and 12 months after the start of treatment. Results: In our population $(n=20)$, an overall increased perception of symptomatic AEs emerged during treatment (statistically significant for abdominal
\end{abstract}

karger@karger.com www.karger.com/etj

2020 European Thyroid Association Published by S. Karger AG, Basel pain, asthenia/fatigue, decreased appetite, and xerostomia). The median EQ-5D index and EQ-VAS scores after 3 months of treatment were lower than at baseline, almost returning to initial values after 12 months. Conclusion: RAI-R TC patients reported a general increase in prevalence and interference on daily activities of symptomatic AEs during therapy with LEN. Self-perceived QoL initially decreased during therapy. However, our data suggest that QoL could be restored after 12 months; this trend might partly reflect the impact of therapy optimisation.

(c) 2020 European Thyroid Association Published by S. Karger AG, Basel

\section{Introduction}

Treatment with lenvatinib (LEN), a multitarget tyrosine kinase inhibitor (TKI), has shown great efficacy in patients with advanced radioactive iodine-refractory (RAI-R) thyroid cancer (TC), both in clinical trials and in a real-life setting [1-4]. Nevertheless, frequent onset of adverse events (AEs) may decrease patients' compliance and, in some cases, lead to discontinuation of therapy [5].

In recent years, growing interest has been dedicated to the study of the quality of life (QoL) perceived by the patients, especially in the oncology setting. Besides the eval- 
uation of traditional endpoints such as efficacy and safety, the assessment of self-perceived mental and physical well-being has been introduced in many clinical trials [6]. Various instruments assessing health-related QoL, such as the European Quality of Life 5 Dimensions (EQ-5D) Questionnaire or the Functional Assessment of Cancer Therapy - General, have been employed to investigate the impact of anticancer therapies [7, 8].

Clinicians generally assess the toxicity of chemotherapeutics using the standardised Common Terminology Criteria for Adverse Events (CTCAE) [9]. However, toxicity occurring between subsequent outpatient visits might not be adequately assessed. The Patient-Reported Outcomes version of the CTCAE (PRO-CTCAE) was conceived with the aim of capturing symptomatic toxicities directly by the patient in order to minimise biases [10]. Ideally, it should allow detecting AEs at an earlier stage, thus simplifying their management. Moreover, it might improve communication between the patient and the physician, strengthening their therapeutic alliance.

The impact of treatment with TKIs on patients' QoL has been extensively investigated both in haematological malignancies and in solid tumours $[11,12]$.

Among patients with locally advanced and/or metastatic RAI-R TC, QoL was assessed during the multicentre, double-blind, randomised, placebo-controlled phase III study evaluating the efficacy and safety of sorafenib (DECISION trial). A small but statistically significant worsening of QoL was observed in the treated patients compared to the placebo group [13]. Conversely, data concerning QoL were not collected in the international phase III study of LEN in advanced RAI-R TC (SELECT trial). A structured assessment of mental and physical well-being during treatment with this TKI in a real-life setting has not yet been reported to our knowledge. Therefore, the aim of our study was to prospectively analyse the impact of LEN on patients' perceived QoL in a group of subjects with advanced RAI-R TC treated at our centre.

\section{Materials and Methods}

We enrolled all the patients with advanced RAI-R papillary, follicular, or poorly differentiated TC who started LEN at our centre between July 31, 2017 and January 31, 2019. Advanced RAI-R TC was defined as locally advanced and/or metastatic disease with no further indication for RAI therapy because of (1) disease progression during therapy with ${ }^{131}$ I, (2) lack of RAI uptake (partial or complete, assessed by ${ }^{131}$ I whole-body scan), or (3) cumulative RAI activity $>600 \mathrm{mCi}$ according to the American Thyroid Association guidelines [14]. We included in this group also patients with advanced disease and an absolute contraindication to ${ }^{131} \mathrm{I}$ therapy after L-thyroxine withdrawal or recombinant human thyrotropin stimulation. Previous treatment with other TKIs was not an exclusion criterion.

All patients had had radiologically confirmed progressive disease in the previous 12 months according to Response Evaluation Criteria in Solid Tumours (RECIST) 1.1 [15]. The drug was started at a daily dose of $24 \mathrm{mg}$, or at a lower dose by clinical judgement (e.g., age, risk of bleeding, or presence of severe comorbidities). AEs during LEN therapy were classified according to the CTCAE, version 4.03 [9]. In case of treatment-related high-grade toxicity, LEN was temporarily interrupted or dose tapered according to the summary of product characteristics of LEN [16]. Significant disease progression or serious toxicity (grade $\geq 3$ according to the CTCAE, not otherwise manageable by symptomatic therapy, dose tapering or brief interruption of LEN) led to a permanent discontinuation of LEN.

At baseline and at the follow-up visits, patients were asked to fill in two different questionnaires in the official Italian version (PRO-CTCAE adapted questionnaire and European Quality of Life 5 Dimensions 3 Levels [EQ-5D-3L] questionnaire) $[7,10]$. The PRO-CTCAE adapted questionnaire investigated the 18 symptomatic AEs experienced by at least $10 \%$ of treated patients in the SELECT trial. For every item, patients could choose an answer to define the presence, the frequency, the severity, and/or the interference with daily activities of a symptomatic $\mathrm{AE}$ during treatment [10]. The EQ-5D-3L questionnaire assesses QoL through the EQ$5 \mathrm{D}$ descriptive system and the EQ visual analogue scale (EQ-VAS); a score (EQ-5D index) can be calculated combining these data into a dedicated algorithm [7].

Patients were asked to fill in the questionnaires anonymously before the consultation with the clinician in the waiting room and to give them back in a closed envelope.

The baseline characteristics of the patients included in the study are summarised using median and interquartile range, mean and standard deviation or numbers and percentages.

To assess treatment efficacy in our population, the disease control rate at the first on-therapy radiological assessment was calculated according to the RECIST criteria; progression-free and overall survival were estimated by the Kaplan-Meier method. The proportions of patients with AEs (all grades) according to clinician's assessment were defined and compared at baseline and at 3,6 , and 12 months after the start of treatment by using Fisher's exact test. The same analysis was performed for the percentage of patients who perceived the symptomatic AEs included in the PRO-CTCAE questionnaire before and during therapy. Furthermore, median values of EQ-5D index and EQ-VAS score at baseline were compared with those at 3,6 , and 12 months after the start of therapy by using the Kruskal-Wallis test. A $p$ value $<0.05$ was considered significant. Statistical analyses were performed with the Stata 15.1 software (StataCorp LP, College Station, TX, USA).

\section{Results}

Twenty consecutive RAI-R TC patients were enrolled in the study. Median age at cancer diagnosis was 58.7 years (interquartile range $=52.5-66.7$ years), while treat- 
Table 1. Patients' characteristics at baseline $(n=20)$

\begin{tabular}{|c|c|c|}
\hline & $n$ & $\%$ \\
\hline \multicolumn{3}{|l|}{ Sex } \\
\hline Female & 10 & 50 \\
\hline Male & 10 & 50 \\
\hline \multicolumn{3}{|l|}{ Histology } \\
\hline Follicular & 9 & 45 \\
\hline Papillary & 7 & 35 \\
\hline Poorly differentiated & 4 & 20 \\
\hline \multicolumn{3}{|l|}{ ECOG performance status } \\
\hline 0 & 9 & 45 \\
\hline 1 & 5 & 25 \\
\hline 2 & 4 & 20 \\
\hline 3 & 1 & 5 \\
\hline 4 & 1 & 5 \\
\hline Locoregional disease & 17 & 85 \\
\hline Distant metastases & 18 & 90 \\
\hline Bone & 10 & 50 \\
\hline Brain & 1 & 5 \\
\hline Lymph nodes & 12 & 60 \\
\hline Lung & 14 & 70 \\
\hline \multicolumn{3}{|l|}{ Surgeries (after thyroidectomy), $n$} \\
\hline 0 & 5 & 25 \\
\hline 1 & 6 & 30 \\
\hline 2 & 6 & 30 \\
\hline 3 & 3 & 15 \\
\hline \multicolumn{3}{|l|}{ Total cumulative radioiodine activity } \\
\hline No ${ }^{131}$ I treatment & 3 & 15 \\
\hline$\leq 100 \mathrm{mCi}$ & 1 & 5 \\
\hline $101-600 \mathrm{mCi}$ & 9 & 45 \\
\hline$>600 \mathrm{mCi}$ & 7 & 35 \\
\hline External beam radiotherapy & 8 & 40 \\
\hline Previous treatment with sorafenib & 3 & 15 \\
\hline \multicolumn{3}{|c|}{ Time from cancer diagnosis to start of LEN therapy } \\
\hline$<1$ year & 3 & 15 \\
\hline $1-4$ years & 6 & 30 \\
\hline$\geq 5$ years & 11 & 55 \\
\hline \multicolumn{3}{|l|}{ Initial daily LEN dose } \\
\hline $24 \mathrm{mg}$ & 6 & 30 \\
\hline $20 \mathrm{mg}$ & 8 & 40 \\
\hline $14 \mathrm{mg}$ & 4 & 20 \\
\hline $10 \mathrm{mg}$ & 2 & 10 \\
\hline
\end{tabular}

ECOG, Eastern Cooperative Oncology Group; LEN, lenvatinib.

ment with LEN was started after a median time of 6.8 years (1.7-12.4 years) from the diagnosis of TC. The patients' main clinical characteristics at baseline are summarised in Table 1.

Six patients started treatment with LEN at a daily dose of $24 \mathrm{mg}$; a lower initial dose was chosen for the remaining population, as displayed in Table 1. Median follow-up was 12.1 months (6.5-19.8 months). The first radiological evaluation was performed after a median time of 3.5 months (2.8-4.2 months) from the start of the treatment; disease control rate was $80 \%$ (7 partial responses and 9 stable diseases). During treatment, progression-free survival at 6 and 12 months was $80.0 \%$ (95\% CI 55.1-91.9\%) and $57.3 \%$ (95\% CI $29.3-77.7 \%$ ), while overall survival at 6 and 12 months was $94.7 \%$ (95\% CI 68.1-99.2\%) and 81.1\% (95\% CI 51.4-93.6\%), respectively.

At least one AE was detected by clinicians in all patients during treatment; AEs of grade $\geq 3$ were registered at least once in $80 \%$ of cases. The proportions of patients with AEs explored in the PRO-CTCAE questionnaire according to medical assessment are displayed in online supplementary Table 1 (for all online suppl. material, see www.karger.com/doi/10.1159/000508186). Furthermore, clinicians detected several AEs of grade $\geq 3$ which were not included in the questionnaires: acute cholecystitis $(n=5)$, gallbladder stones $(n=2)$, congestive heart failure $(n=1)$, aortic dissection $(n=1)$, sepsis $(n=1)$, pneumonitis $(n=1)$, and pulmonary embolism $(n=1)$.

In order to manage toxicity during treatment, the LEN dose was reduced or temporarily interrupted in 80 and $85 \%$ of patients, respectively, resulting in a mean dose of $13.9 \mathrm{mg}$ ( $\pm 5.5 \mathrm{mg}$ ) per day. The first dose reduction was necessary after a median time of 2.7 months (1.6-4.3 months), while the first drug interruption occurred after a median time of 2.8 months (1.9-3.7 months). Treatment with LEN was permanently discontinued in 2 subjects for clinically significant progressive disease and in 2 cases for severe toxicity (in 1 patient for asthenia, gastrointestinal symptoms, and recurrent pneumonitis, and in the other one for severe congestive heart failure).

A general increase in the perception of the AEs included in the PRO-CTCAE questionnaires was initially registered during treatment when compared to baseline, reaching statistical significance in case of abdominal pain (severity, $p=0.02$; interference, $p<0.01$ ), decreased appetite (severity, $p=0.04$ ), xerostomia (severity, $p=0.02$ ), and asthenia/fatigue (interference, $p<0.01$ ) (Fig. 1). Online supplementary Table 2 shows the percentage of patients perceiving the AEs included in the PRO-CTCAE questionnaires before and during therapy.

The 12-month assessment was available for half of the initial population: within the first year of treatment, 2 patients died and 3 patients permanently discontinued the drug (in 2 cases for progressive disease and in 1 case for severe toxicity); moreover, in 5 patients the follow-up was $>6$ months but $<12$ months. The proportion of patients reporting symptomatic AEs after 12 months of treatment 


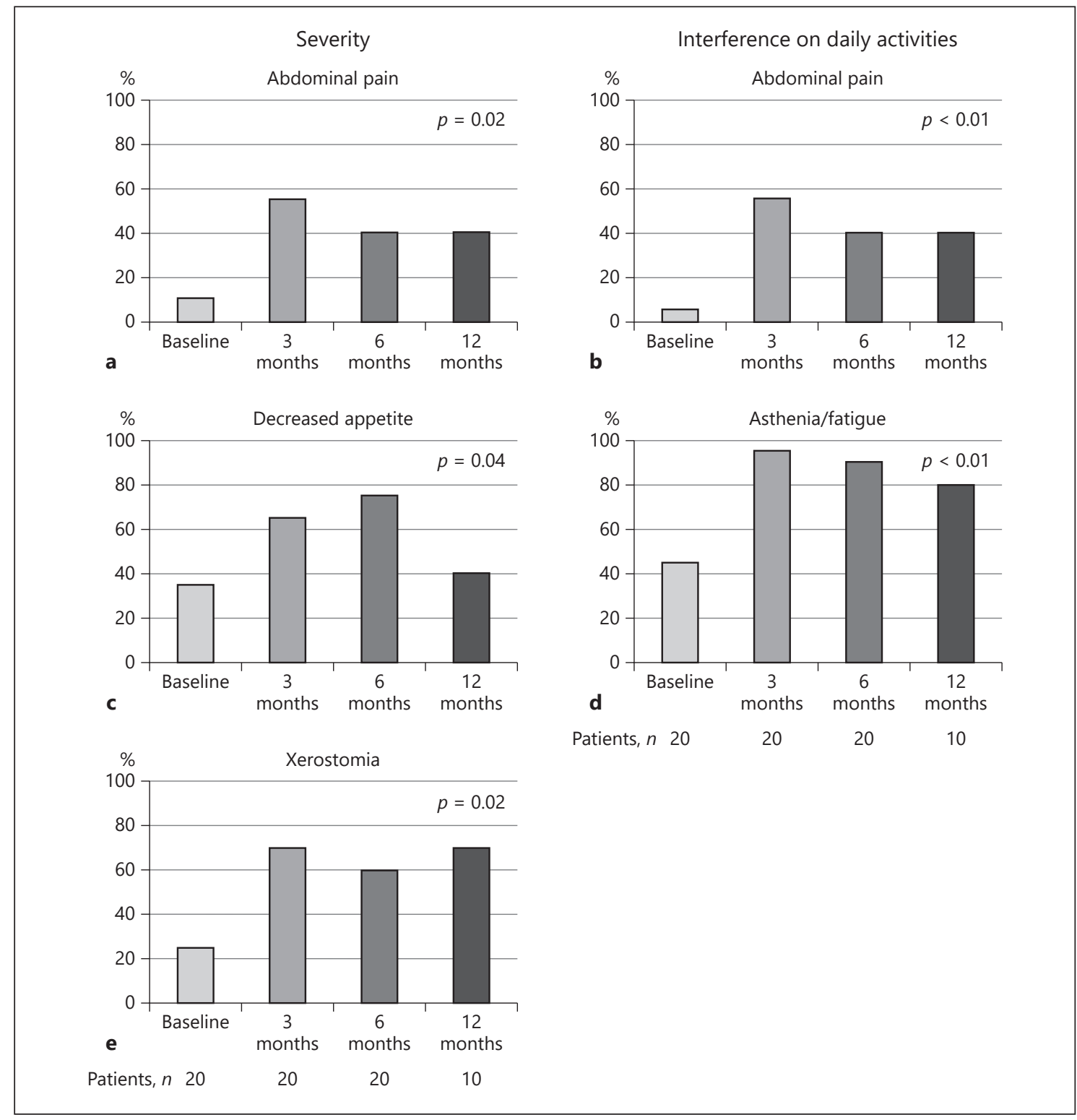

Fig. 1. Percentage of patients perceiving any degree of severity (at least mild) and interference on daily activities of AEs at baseline and during treatment with LEN (according to the PRO-CTCAE questionnaires. a Abdominal pain (severity). b Abdominal pain (interference). c Decreased appetite (severity). d Asthenia/fatigue (interference). e Xerostomia (severity). AEs, adverse events; LEN, lenvatinib; PRO-CTCAE, Patient-Reported Outcomes version of the Common Terminology Criteria for Adverse Events.

was generally lower than the one reported after 3 months of therapy.

In comparison to baseline, a decreased EQ-5D index was registered in all but 1 case during treatment, while a lower EQ-VAS was observed in 17 out of 20 patients. Among the 10 patients who filled in the 12-month questionnaire, the EQ-5D index and the EQ-VAS score were equal or higher than at baseline in 7 and 8 cases, respectively.

The median EQ-5D index before the start of treatment was higher than after 3 and 6 months of therapy, but similar to that observed after 12 months of treatment ( $p=$ $0.04)$; an analogous trend was detected for the median EQ-VAS score (0.02), as shown in Figure 2. 

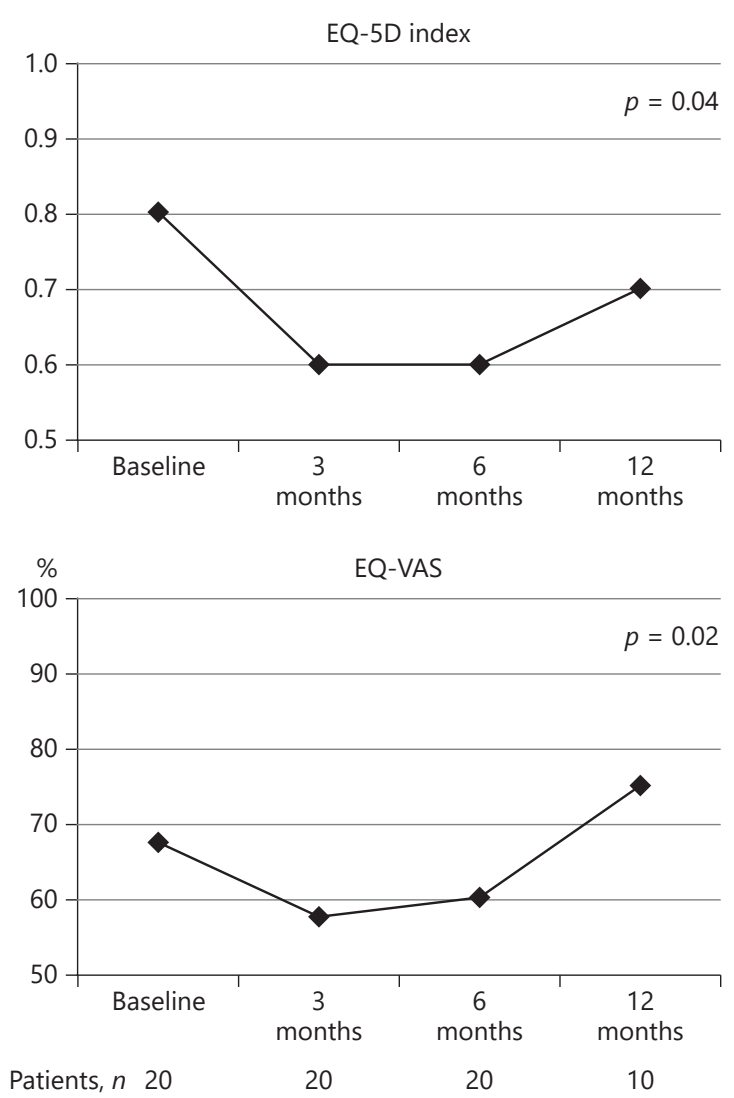

Fig. 2. Median EQ-5D index and EQ-VAS before the start of therapy and during treatment with LEN. EQ-5D, European Quality of Life 5 Dimensions; EQ-VAS, European Quality of Life visual analogue scale; LEN, lenvatinib.

\section{Discussion}

In our population, a general increase in prevalence and interference of symptomatic AEs on daily activities was reported during therapy with LEN. Self-perceived QoL initially decreased during therapy, but it could ameliorate after 12 months of therapy.

LEN is considered a first-line systemic treatment for patients with RAI-R TC and progressive disease according to the most recent guidelines [17]. Neither the phase III SELECT trial nor the main multicentre retrospective observational studies included the evaluation of patients' perceived QoL.

In our population, only 3 patients had already been treated with another TKI (sorafenib in all cases) before starting therapy with LEN; therefore, the results of our analysis mostly reflect the real impact of LEN treatment rather than the cumulative effect of different TKIs.

The percentage of patients with impaired clinical condition at baseline exceeded the proportion reported both in the SELECT trial and in the most relevant real-life studies; it implies the need of a personalised strategy from the very early phases of therapy.

Disease control rate at first radiological evaluation as well as progression-free and overall survival at 6 months of therapy confirm the clinical efficacy of LEN in terms of disease control, similar to other real-life studies, also in our population of patients with generally more advanced disease $[2,3]$.

At least one AE was recorded in all subjects, and severe AEs were registered in more than two-thirds of our patients. A statistically significant worsening of asthenia, diarrhoea, hand-foot syndrome, and xerostomia during treatment was detected at our evaluations when compared to baseline. These findings confirm the consistent toxic profile of this drug already reported in similar studies. Interestingly, constitutional symptoms (e.g., asthenia, arthralgia, myalgia) were detected in more than one-third of the population at baseline, reflecting the presence of impaired clinical conditions even before the onset of therapy.

During treatment, our patients felt an increased frequency, severity, and interference on daily activities of the symptomatic AEs included in the PRO-CTCAE questionnaires, especially in the first months of therapy, independently from the initial dose. These changes reached statistical significance in case of abdominal pain (severity and interference), decreased appetite (severity), xerostomia (severity), and asthenia/fatigue (interference). The proportion of patients experiencing AEs at 12 months did not further increase.

The temporal trend of AE occurrence is similar to that recently described for weight loss during LEN therapy [18]. Taking into account that most patients underwent reduction and/or transient interruption of the drug within the first 6 months, it could be speculated that the positive trend in the perception of AEs during therapy might partly reflect the impact of therapy optimisation in the management of drug toxicity. However, no solid conclusion can be drawn from these limited data, especially considering that a filled-in 12-month questionnaire was available only for half of the population.

It could also be hypothesised that the initial tumour response to LEN therapy might have played a role in the self-perception of QoL, especially in patients with symptoms due to the tumour burden at baseline. Moreover, 
both the initial LEN dose and the Eastern Cooperative Oncology Group (ECOG) status could have influenced patients' QoL. In our cohort, we were not able to find a significant association between these factors and QoL changes, due to the small sample and the presence of several confounding factors in a real-life setting (i.e., choice of lower LEN starting dose in patients with poor baseline ECOG status and vice versa).

Both the median EQ-5D index and the EQ-VAS score before the start of treatment were significantly higher than the values registered after 3 and 6 months of therapy. These data reflect an initial reduction in self-perceived QoL after the start of therapy, which needs to be recognised and properly managed in order to maintain and enforce patients' adherence to treatment. However, these QoL parameters became similar to the basal ones after 12 months of treatment. In our population, patient-tailored modulation of treatment - together with the start of symptomatic therapy - might have played a role in the improvement of patients' well-being during therapy, although specific data supporting this hypothesis are lacking.

It could be argued that our analysis might be biased by the fact that only patients who tolerated the drug could continue treatment and fill in all questionnaires. However, in our population only 2 patient discontinued therapy with LEN for unmanageable toxicity: one between 6 and 12 months of therapy and the other one after 24 months of therapy.

The small sample size and the limited follow-up represent the main limitations of this pilot analysis of QoL, which needs to be explored in studies with larger cohorts of patients and with a prolonged duration. Also, the lack of a control group (patients with advanced RAI-R differentiated TC not treated with LEN) represents a drawback of this analysis. Nevertheless, the prospective design of the study limited the potential biases of our research. Moreover, RAI-R differentiated TC is a relatively rare condition and LEN has been approved for the management of this disease only for a few years.

The main strength of this study is that, for the first time, we prospectively gathered data focusing on self-perceived QoL in a group of RAI-R TC patients treated with LEN homogeneously managed at a single referral centre. Our findings draw attention to the patients' perspective and help clinicians to gain increasing awareness in the real impact of the drug on physical and mental well-being of these subjects. Patients should be informed about the possibility of perceiving several symptomatic AEs from the very early phases of treatment. The introduction of self-administered questionnaires for the evaluation of drug toxicity in a real-life setting might be helpful for clinicians, since it records mild symptoms that could not emerge during the outpatient visit. The early detection of AEs might improve patients' clinical outcome and preserve compliance and adherence to this lifelong treatment.

In conclusion, our study showed that a relevant toxicity was recorded by clinicians during treatment with LEN for RAI-R TC. Patients reported a general increase in frequency, severity, and interference on daily activities of symptomatic AEs on therapy when compared to baseline. Self-perceived QoL decreased during the first months of therapy; nevertheless, patients' well-being seemed not to be worsened by the cumulative toxicity of the drug over time and QoL was restored after 12 months of therapy, perhaps reflecting, at least in part, the consequence of the proper management of the drug-related AEs. We believe that the early detection of self-perceived AEs as well as changes in QoL might improve patients' clinical outcome and preserve compliance to this lifelong treatment. Further investigations are needed to better understand the real global impact of LEN treatment on TC patients, especially in the long term.

\section{Statement of Ethics}

All procedures were performed in accordance with the ethical standards of the institutional research committee and with the 1964 Helsinki Declaration and its later amendments or comparable ethical standards. The administration of the questionnaires was approved by our local ethics committee, and all patients provided informed consent to participate in the study.

\section{Conflict of Interest Statement}

There is no conflict of interest that could be perceived as prejudicing the impartiality of the research reported.

\section{Funding Sources}

This research did not receive any specific grant from any funding agency in the public, commercial, or not-for-profit sector.

\section{Author Contributions}

A. Nervo: design of the study, data collection and analyses, data interpretation, and writing of the manuscript draft. A. Ragni, V. Marica: data collection and analyses, and writing of the manuscript draft. E. Migliore: statistical analyses and data interpretation. A. Piovesan, M. Gallo, E. Arvat: data interpretation and revision of the final paper.
Nervo/Ragni/Piovesan/Marica/Migliore/ Gallo/Arvat 


\section{References}

1 Schlumberger M, Tahara M, Wirth LJ, Robinson B, Brose MS, Elisei R, et al. Lenvatinib versus placebo in radioiodine-refractory thyroid cancer. N Engl J Med. 2015 Feb;372(7):62130.

2 Berdelou A, Borget I, Godbert Y, Nguyen T, Garcia ME, Chougnet $\mathrm{CN}$, et al. Lenvatinib for the treatment of radioiodine-refractory thyroid cancer in real-life practice. Thyroid. 2017 doi: 10.1089/thy.2017.0205 [Epub ahead of print].

3 Locati LD, Piovesan A, Durante C, Bregni M, Castagna MG, Zovato S, et al. Real-world efficacy and safety of lenvatinib: data from a compassionate use in the treatment of radioactive iodine-refractory differentiated thyroid cancer patients in Italy. Eur J Cancer. 2019 Sep;118:35-40.

4 Nervo A, Gallo M, Samà MT, Felicetti F, Alfano M, Migliore E, et al. Lenvatinib in Advanced Radioiodine-refractory Thyroid Cancer: A Snapshot of Real-life Clinical Practice. Anticancer Res. 2018 Mar;38(3):1643-9.

5 Haddad RI, Schlumberger M, Wirth LJ, Sherman EJ, Shah MH, Robinson B, et al. Incidence and timing of common adverse events in Lenvatinib-treated patients from the SELECT trial and their association with survival outcomes. Endocrine. 2017 Apr;56(1):121-8.

6 Fallowfield L. Quality of life: a new perspective for cancer patients. Nat Rev Cancer. 2002 Nov;2(11):873-9.
7 Rabin R, de Charro F. EQ-5D: a measure of health status from the EuroQol Group. Ann Med. 2001 Jul;33(5):337-43.

8 Cella DF, Tulsky DS, Gray G, Sarafian B, Linn E, Bonomi A, et al. The Functional Assessment of Cancer Therapy scale: development and validation of the general measure. J Clin Oncol. 1993 Mar;11(3):570-9.

9 https://evs.nci.nih.gov/ftp1/CTCAE/CTCAE_4.03/CTCAE_4.03_2010-06-14 QuickReference_5x7.pdf [accessed December 4, 2019].

10 https://healthcaredelivery.cancer.gov/proctcae [accessed December 4, 2019].

11 Efficace F, Cannella L. The value of quality of life assessment in chronic myeloid leukemia patients receiving tyrosine kinase inhibitors. Hematology Am Soc Hematol Educ Program. 2016 Dec;2016(1):170-9.

12 Li D, Sedano S, Allen R, Gong J, Cho M, Sharma S. Current Treatment Landscape for Advanced Hepatocellular Carcinoma: Patient Outcomes and the Impact on Quality of Life. Cancers (Basel). 2019 Jun;11(6):E841.

13 Schlumberger M, Jarzab B, Elisei R, Siena S, Bastholt L, de la Fouchardiere C, et al. Phase III randomized, double-blinded, placebo controlled trial of sorafenib in locally advanced or metastatic patients with radioactive iodine (RAI)-refractory differentiated thyroid cancer (DTC) - exploratory analyses of patientreported outcomes (abstract). San Juan: 83rd Annual Meeting of the American Thyroid Association; 2013
14 Haugen BR, Alexander EK, Bible KC, Doherty GM, Mandel SJ, Nikiforov YE, et al. 2015 American Thyroid Association Management Guidelines for Adult Patients with Thyroid Nodules and Differentiated Thyroid Cancer: The American Thyroid Association Guidelines Task Force on Thyroid Nodules and Differentiated Thyroid Cancer. Thyroid. 2016 Jan;26(1):1-133.

15 Eisenhauer EA, Therasse P, Bogaerts J, Schwartz LH, Sargent D, Ford R, et al. New response evaluation criteria in solid tumours: revised RECIST guideline (version 1.1). Eur J Cancer. 2009 Jan;45(2):228-47.

16 https://www.ema.europa.eu/en/documents/ product-information/lenvima-epar-productinformation_en.pdf [accessed December 4, 2019].

17 Fugazzola L, Elisei R, Fuhrer D, Jarzab B, Leboulleux S, Newbold K, et al. 2019 European Thyroid Association Guidelines for the Treatment and Follow-Up of Advanced Radioiodine-Refractory Thyroid Cancer. Eur Thyroid J. 2019 Oct;8(5):227-45.

18 De Leo S, Colombo C, Di Stefano M, Dubini A, Cozzi S, Persani L, et al. Body Composition and Leptin/Ghrelin Levels during Lenvatinib for Thyroid Cancer. Eur Thyroid J. 2020 Jan; 9(1):1-10. 